\title{
Objective Olfactory Evaluation of Self-reported Loss of Smell in a Case Series of 86 COVID-19 Patients
}

\author{
Jerome R. Lechien, MD, PhD, MS ${ }^{1}$ \\ ${ }^{1}$ Universite de Mons
}

May 7, 2020

\begin{abstract}
Objective : To investigate olfactory dysfunction (OD) in patients with mild COVID-19 through patient-reported outcome questionnaires and objective psychophysical testing.

Methods : COVID-19 patients with self-reported sudden-onset OD were recruited. Epidemiological and clinical data were collected. Nasal complaints were evaluated with the sino-nasal outcome-22 (SNOT-22). Subjective olfactory and gustatory status was evaluated with the National Health and Nutrition Examination Survey (NHNES). Objective OD was evaluated using psychophysical tests.

Results : Eighty-six patients completed the study. The most common symptoms were fatigue (72.9\%), headache (60.0\%), nasal obstruction $(58.6 \%)$ and postnasal drip (48.6\%). Total loss of smell was self-reported by $61.4 \%$ of patients. Objective olfactory testings identified 41 anosmic (47.7\%), 12 hyposmic (14.0\%), and 33 normosmic (38.3\%) patients. There was no correlation between the objective test results and subjective reports of nasal obstruction or postnasal drip.
\end{abstract}

Conclusion : A significant proportion of COVID-19 patients reporting OD do not have OD on objective testing.

Jerome R. Lechien, MD, PhD, MS ${ }^{1-4}$, Pierre Cabaraux MD ${ }^{1-5}$, Carlos M. Chiesa-Estomba MD, MS ${ }^{1,6}$, Mohamad Khalife $\mathrm{MD}^{1,7}$, Stéphane Hans, MD, $\mathrm{PhD}, \mathrm{MS}^{1,3}$, Christian Calvo-Henriquez, $\mathrm{MD}^{1,8}$, Delphine Martiny, PharmD, $\mathrm{PhD}^{9}$, Fabrice Journe, $\mathrm{PhD}^{1,2}$, Leigh Sowerby, MD, MHM, FRCSC ${ }^{1,10}$, Sven Saussez, $\mathrm{MD}, \mathrm{PhD}^{2,4,7}$

\section{Institutions:}

1. COVID-19 Task Force of the Young-Otolaryngologists of the International Federations of Oto-rhinolaryngological Societies (YO-IFOS).

2. Department of Human Anatomy and Experimental Oncology, Faculty of Medicine, UMONS Research Institute for Health Sciences and Technology, University of Mons (UMons), Mons, Belgium

3. Department of Otolaryngology-Head \& Neck Surgery, Foch Hospital, School of Medicine, UFR Simone Veil, Université Versailles Saint-Quentin-en-Yvelines (Paris Saclay University), Paris, France.

4. Department of Otorhinolaryngology and Head and Neck Surgery, CHU de Bruxelles, CHU Saint-Pierre, School of Medicine, Université Libre de Bruxelles, Brussels, Belgium.

5. Department of Neurology, CHU de Charleroi, Charleroi, Belgium.

6. Department of Otorhinolaryngology-Head \& Neck Surgery, Hospital Universitario Donostia, San Sebastian, Spain.

7. Department of Head and neck Surgery, EpiCURA Hospital, Hornu, Belgium.

8. Department of otolaryngology-Hospital Complex of Santiago de Compostela, Santiago de Compostela, Spain.

9. Department of Microbiology, Laboratoire Hospitalier Universitaire de Bruxelles - Universitair Laboratorium Brussel (LHUB-ULB), Brussels, Belgium; Faculté de Médecine et Pharmacie, Université de Mons (UMONS), Mons, Belgium. 
10. Department of Otolaryngology - Head and Neck Surgery, University of Western Ontario, London, Ontario, Canada.

Running title : Coronavirus and Olfaction.

Key words : COVID-19; anosmia; olfaction; olfactory; smell; evaluation; taste; coronavirus.

\section{Author contribution:}

Study concept and design: Lechien, Cabaraux, Chiesa, Calvo-Henriquez, Saussez, Hans, Khalife.

Acquisition, analysis, or interpretation of data : Cabaraux, Lechien, Saussez, Journe, Khalife, Martiny, Sowerby.

Drafting of the manuscript: Lechien, Saussez, Sowerby.

Critical revision of the manuscript for important intellectual content: Sowerby, Saussez, Chiesa, CalvoHenriquez, Hans, Journe, Khalife, Carabaux.

\section{Correspondence to :}

Dr. Jay R. Lechien, M.D., Ph.D., M.S.

Department of Otorhinolaryngology and Head and Neck Surgery, Foch Hospital, School of Medicine, UFR Simone Veil, Université Versailles Saint-Quentin-en-Yvelines (Paris Saclay University), Paris, France.

Email: Jerome.Lechien@umons.ac.be

Telephone: +32 65373584

\section{Abstract :}

Objective : To investigate olfactory dysfunction (OD) in patients with mild COVID-19 through patientreported outcome questionnaires and objective psychophysical testing.

Methods : COVID-19 patients with self-reported sudden-onset OD were recruited. Epidemiological and clinical data were collected. Nasal complaints were evaluated with the sino-nasal outcome-22 (SNOT-22). Subjective olfactory and gustatory status was evaluated with the National Health and Nutrition Examination Survey (NHNES). Objective OD was evaluated using psychophysical tests.

Results : Eighty-six patients completed the study. The most common symptoms were fatigue $(72.9 \%)$, headache $(60.0 \%)$, nasal obstruction (58.6\%) and postnasal drip (48.6\%). Total loss of smell was self-reported by $61.4 \%$ of patients. Objective olfactory testings identified 41 anosmic (47.7\%), 12 hyposmic (14.0\%), and 33 normosmic (38.3\%) patients. There was no correlation between the objective test results and subjective reports of nasal obstruction or postnasal drip.

Conclusion : A significant proportion of COVID-19 patients reporting OD do not have OD on objective testing.

\section{Introduction:}

Since the onset of the coronavirus disease 2019 (COVID-19) pandemic in Europe, many otolaryngologists have reported patients with a sudden loss of smell. ${ }^{1,2}$ Olfactory dysfunction is rapidly becoming a key symptom of COVID-19, with more than $66 \%$ of patients in Europe and U.S reporting some degree of hyposmia. ${ }^{1,3-6}$ The loss of smell has been reported to occur before (11.8\%), after $(65.4 \%)$ or at the same time $(22.8 \%)$ as the onset of other general or otolaryngological symptoms. ${ }^{1}$ Knowledge around the relationship between olfactory dysfunction and COVID-19 is rapidly evolving. Recently, Yan et al . shown that anosmia seems to be associated with a milder clinical course in patients with COVID-19. ${ }^{6}$ Moein et al . suggested that $98 \%$ of 60 Iranian COVID-19 patients exhibited some olfactory dysfunction on objective testing; only $35 \%$ of these patients were aware of hyposmia/anosmia before testing. ${ }^{7}$ The nuances around olfaction in COVID-19 
appear to be associated with different clinical parameters than other symptoms, and, consequently, warrant further investigation.

The objective of this study was to investigate the olfactory dysfunction of COVID-19 patients with subjective validated patient-reported outcome questionnaires and objective psychophysical testing.

\section{Methods:}

The study protocol was approved by the ethics committee of the Jules Bordet Institute (Central Ethics Committee, IJB-0M011-3137). Patients were invited to participate and informed consent was obtained once inclusion criteria were met.

\section{Setting}

Adult patients with confirmed COVID-19 and self-reported sudden-onset olfactory dysfunction were recruited through a public call from the Department of Anatomy of the University of Mons (Mons, Belgium). To be included, patients had to be not currently hospitalized (mild-to-moderate patients). The diagnosis of COVID-19 infection was based on the WHO interim guidance and symptoms of disease [8]. Individuals with self-reported sudden olfactory dysfunction and a clinical history suggestive of COVID-19 were invited to participate. A nasopharyngeal swab was performed to identify severe acute respiratory coronavirus-2 (SARS-CoV-2) via reverse transcription polymerase chain reaction (RT-PCR) for patient with symptom duration $<14$ days. In case of negative RT-PCR, serology for IgG and IgM to SARS-CoV-2 was realized. For patients with symptom duration [?]14 days, physicians performed serology (Figure 1). Only patients with a RT-PCR positive test or with positive IgG or IgM were included. Patients with a history of olfactory dysfunction before the pandemic, history of nasal surgery, chronic rhinosinusitis, head \& neck trauma, or degenerative neurological disease were excluded from the study.

\section{COVID-19 diagnosis}

The RT-PCR was performed by an experienced microbiologist (D.M.) in the LHUB-ULB laboratory of Brussels (Laboratoire Hospitalier Universitaire de Bruxelles, Belgium). Viral RNA extraction was performed

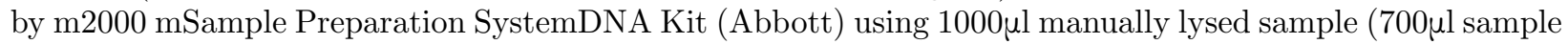
$+800 \mu l$ lysis buffer from kit) eluted in 90ul elution buffer. A qRT-PCR internal control was added at each extraction. qRT-PCR was performed using $10 \mu \mathrm{l}$ of extracted sample in the RealStar ${ }^{\circledR}$ SARS-CoV-2 RT-PCR Kit from Altona-diagnostics with a cut-off set at 40 cycle threshold $(\mathrm{Ct})$.

Patients with a negative RT-PCR benefited from a serological test (Zentech, University of Liege Lab, Liege, Belgium) to determine whether or not they have been exposed to SARS-Cov-2.

\section{Epidemiological $\&$ Clinical outcomes}

To minimize the risk of exposure for study personnel, the clinical and epidemiological characteristics of patients were electronically collected via an online questionnaire developed with Professional Survey Monkey $\mathbb{R}$ (San Mateo, California, USA). Demographic data including gender, age and ethnicity, as well as patient comorbidities and medications were collected.

\section{General and Otolaryngological Symptoms}

The following general and ear, nose, and throat symptoms were collected and rated (from $0=$ no symptom to $4=$ severe symptom): cough, chest pain, dyspnea, headache, fever, fatigue, loss of appetite, myalgia, arthralgia, nausea, vomiting, diarrhea, excessive sticky sputum, skin manifestations (urticaria), conjunctivitis, nasal obstruction, postnasal drip, rhinorrhea, sore throat, facial pain, ear pain, dysphagia, dysphonia and dysgeusia. Dysgeusia was defined as the impairment of salty, sweet, bitter and sour.

\section{Patient-reported outcome questionnaires}

The impact of COVID-19 on sinonasal symptoms was evaluated through the French version of the sino-nasal outcome test-22 (SNOT-22), ${ }^{9}$ a validated patient-reported outcome questionnaire from the original U.S. 
20-item version. ${ }^{10}$

The impact of olfactory dysfunction on quality of life was assessed through the short version of the Questionnaire of Olfactory Disorders-Negative Statements (sQOD-NS). ${ }^{11}$ sQOD-NS is a 7-item patient-reported outcome questionnaire. Patients rated the item proposition from 0 (agree) to 3 (disagree) with total score ranging from 0 (significant impact of olfactory dysfunction on QoL) to 21 (no impact on QoL). Authors used sQOD-NS for its ease of completion.

The olfactory and gustatory questions were based on the smell and taste component of the National Health and Nutrition Examination Survey (NHNES). ${ }^{12}$ NHNES is a population survey that continuously monitors the health of adult citizens in the U.S. through a nationally representative sample of 5,000 persons on a yearly basis. ${ }^{12}$ The questions have been selected to characterize the variation, timing and associated-symptoms of both olfactory and gustatory dysfunction.

\section{Psychophysical Olfactory Evaluation}

The psychophysical olfactory evaluations were performed using the identification sniffin' sticks test (Medisense, Groningen, The Netherlands), which is a validated objective test of olfactory dysfunction. ${ }^{13}$ A total of 16 scents were presented via a pen device to patients for 3 seconds followed by a forced choice from 4 given options with a total possible score of 16 points. According to the results, patients were classified as normosmic (score between 12-16), hyposmic (score between 9-11) or anosmic (score 8 or below).

\section{Statistical Analyses}

Statistical analyses were performed using the Statistical Package for the Social Sciences for Windows (SPSS version 22,0; IBM Corp, Armonk, NY, USA). The relationship between clinical and olfactory outcomes was analyzed through non-parametric test using Spearman correlation for scale data, Khi2 test for ordinal data and Mann-Whitney or Kruskal-Wallis test for categorized data. We investigated all potential associations between nasal complaints (nasal obstruction, rhinorrhea, postnasal drip) and the occurrence of olfactory disorder (sniffin'stick test). A level of significance of $\mathrm{p}<0.05$ was used.

\section{Results:}

A total of 86 patients were eligible and completed the study (Figure 1). There were 56 females (65.1\%) and 30 males $(34.9 \%)$. The mean age was $42 \pm 12$ years. The majority of patients were Caucasian. Reflux, asthma and allergic rhinitis were the most common comorbidities (Table 1). Non-smokers accounted for $90 \%$ of the cohort.

Clinical outcomes based on the general questionnaire.

The most common general symptoms developed over the clinical course were fatigue (72.9\%), headache $(60.0 \%)$, cough $\left(48.6 \%\right.$ ) and myalgia (42.9\%) (Table 2). Fever, defined as a body temperature $>38 \mathrm{C}^{\circ}$, was only reported by $8.6 \%$ of patients. Asthenia was the most commonly reported severe general symptom. The most common otolaryngological symptoms were nasal obstruction ( $58.6 \%$ ), postnasal drip (48.6\%), and dysgeusia (47.1\%). Dysgeusia was considered the most severe otolaryngological symptom by half of the surveyed patients (Table 3 ).

\section{Patient Reported Outcome Questionnaire of Olfactory \& Gustatory Function}

According to the NHNES questions, $61.4 \%$ of patients described their olfactory disorder as total loss of smell at the onset of the disease, while the remainder reported partial loss. Cacosmia and phantosmia occurred in $34 \%$ and $20 \%$ patients, respectively. The mean scores of SNOT-22 and sQOD-NS are reported in Table 4.

Regarding gustatory dysfunction, $51 \%$ of patients reported taste disorders with abnormal sensations of salty, sweet, bitter and sour. The aroma perception was completely or partly lost in $42 \%$ and $32 \%$, respectively, while $12 \%$ reported distortion of aroma.

Psychophysical Olfactory Evaluations 
The mean score of Sniffin' Stick testing was $9 \pm 4$. Among the 86 patients, 41 (48\%) and 12 (14\%) patients were anosmic and hyposmic, respectively. A total of $33(38 \%)$ patients who reported loss of smell were objectively normosmic. In the anosmic group, 26 (78.8\%) patients reported total loss of smell. In the second group, 8 hyposmic individuals $(88.9 \%)$ reported total loss of smell (Table 5).

The mean durations of olfactory dysfunction at the time of the evaluations were $17 \pm 11$ days and $18 \pm$ 11 days for anosmic and hyposmic patients, respectively. The mean duration of olfactory dysfunction of normosmic patients was $17 \pm 11$ days (Table 5).

Eleven patients realized sniffin'stick test twice (one week apart). Among these 11 patients, 9 were anosmic, 1 hyposmic and 1 normosmic at the first evaluation. From the first to the second visit (1 week later), the sniffin'stick test values improved in 5 patients ( 1 became hyposmic and 4 normosmic individuals) of the 9 anosmic patients of the first visit.

\section{Subgroup Analysis 83 Relationship between Outcomes}

The nasal obstruction was not significantly associated with the development of olfactory dysfunction. Among the anosmic group, $60.1 \%$ of patients did not suffer from nasal obstruction (Table 5). There was no significant association between the results of the sniffin' stick tests and the occurrence/severity of the following complaints: nasal obstruction and postnasal drip.

\section{Discussion:}

The involvement of COVID-19 in the development of olfactory and gustatory dysfunctions seems obvious. However, the characterization of the pathophysiological mechanisms underlying the olfactory dysfunction remains challenging regarding the risk of contamination. In this study, we have performed both subjective and objective olfactory evaluations in COVID-19 patients through online patient-reported outcome questionnaires and individual objective psychophysical testings. Interestingly, $38 \%$ of patients with self-reported olfactory dysfunction had normal olfactory testing at the sniffin'stick test.

The mismatch between the self-reported loss of smell and the anosmia regarding psychophysical testings has already been suggested in a recent Italian study where a few COVID-19 patients, who self-reported loss of smell, were objectively anosmic. ${ }^{14}$ Thus, the prevalence of olfactory dysfunction related to COVID-19 would be overestimated in the epidemiological studies where the loss of smell was based on subjective reports.

Another important finding of this study is the non-significant relationship between symptoms of nasal inflammation and objective olfactory dysfunction. In most cases of olfactory dysfunction occurring in viral infections, the olfactory disorder is related to the inflammatory reaction of the mucosa, leading to nasal obstruction, rhinorrhea and postnasal drip. In some cases, the olfactory dysfunction appeared to be related to other mechanisms, such as a neural spread of the virus into the neuroepithelium and the olfactory bulb. In 2007, Suzuki et al . demonstrated that coronavirus may be detected in the nasal discharge of patients with olfactory dysfunction. ${ }^{15}$ In this study, some patients had normal acoustic rhinometry, suggesting that nasal inflammation and related obstruction were not the only etiological factors underlying the olfactory dysfunction in viral infection. Netland et al . demonstrated on transgenic mice expressing the SARS-CoV receptor (human angiotensinconverting enzyme 2) that SARS-CoV may enter the brain through the olfactory bulb, leading to rapid transneuronal spread. ${ }^{16}$ The neurotropism of the COVID-19 is not new and would be associated with other symptoms and findings. For example, the virus spread into the central nervous system is currently suspected to play a key role in respiratory failure through an effect on the medullary cardiorespiratory center. ${ }^{17}$ Similarly, the existence of different patterns of gustatory and olfactory recoveries would be explained by selective neurological impairments. ${ }^{1}$ In other words, and suggested by the aroma and gustatory outcomes, the loss of taste would be not a retro-olfactory disorder in some patients. Future experimental and clinical studies are needed to better understand the pathophysiological mechanisms underlying the development of olfactory and gustatory dysfunctions. These studies would associate patientreported outcome questionnaires, psychophysical olfactory evaluations, fiberoptic examinations, and imaging or neurophysiological assessments. 
The main limitation of the present study is the heterogeneity between patients about the duration of the olfactory dysfunction. However, it is complicated to recruit patients at the first day of the olfactory disorder for many reasons. First, many patients have other troublesome symptoms (e.g. fatigue, myalgia, arthralgia), which may limit the realization of the tests. Second, the recruitment of patients at the first day of the olfactory dysfunction involved a continuous communication to recruit these patients. In practice, it is complicated to communicate with the general public every day for a scientific study. The lack of full objective methods to assess olfaction may be considered as another weakness. In this study, we decided to use the Identification sniffin'sticks test (16 items) for practical and ethical reasons. This test may be performed quickly, which is important to reduce the risk of potential contamination of caregivers.

\section{Conclusion:}

Only $62 \%$ of COVID-19 patients with self-reported olfactory dysfunction have anosmia or hyposmia on objective psychophysical olfactory evaluation. Interestingly, the majority of those with confirmed objective olfactory dysfunction did not have nasal inflammatory symptoms, supporting the need of future clinical and experimental studies to clarify the pathophysiological mechanisms underlying the development of anosmia in COVID-19.

Funding : FRMH Funding.

Conflict of interest statement : The authors have no conflicts of interest

\section{Acknowledgments: - .}

\section{References:}

1. Lechien JR, Chiesa-Estomba CM, De Siati DR, et al. Olfactory and gustatory dysfunctions as a clinical presentation of mild-to-moderate forms of the coronavirus disease (COVID-19): a multicenter European study. Eur Arch Otorhinolaryngol . 2020. doi: 10.1007/s00405-020-05965-1. 2. Hopkins C, Surda P, Kumar N. Presentation of new onset anosmia during the COVID-19 pandemic. Rhinology . 2020. doi: 10.4193/Rhin20.116.

3. Lechien JR, Chiesa-Estomba CM, Place S, et al (in press). Clinical and Epidemiological Characteristics of 1,420 European Patients with mild-to-moderate Coronavirus Disease 2019. In press, J Int Med . 2020

4. Kaye R, Chang DW, Kazahaya K, Brereton J, Denneny III JC. COVID-19 Anosmia Reporting Tool: Initial Findings. Accepted for publication in Otolaryngol Head Neck Surg . 2020.

5. Beltrán-Corbellini Á, Chico-García JL, Martínez-Poles J, et al. Acute-onset smell and taste disorders in the context of Covid-19: a pilot multicenter PCR-based case-control study. Eur J Neurol . 2020 Apr 22. doi: 10.1111/ene. 14273

6. Yan CH, Faraji F, Prajapati DP, Ostrander BT, DeConde AS. Self-reported olfactory loss associates with outpatient clinical course in Covid-19. Int Forum Allergy Rhinol. 2020 Apr 24. doi: 10.1002/alr.22592.

7. Moein ST, Hashemian SMR, Mansourafshar B, Khorram-Tousi A, Tabarsi P, Doty RL. Smell dysfunction: a biomarker for COVID-19. Int Forum Allergy Rhinol . 2020. doi: 10.1002/alr.22587.

8. World Health Organization. Clinical management of severe acute respiratory infection when novel coronavirus (2019- $\mathrm{nCoV})$ infection is suspected: interim guidance. January 28, 2020 https://www.who.int/docs/default-source/coronaviruse/clinical-management-of-novel-cov.pdf

9. de Dorlodot C, Horoi M, Lefebvre P, Collet S, Bertrand B, Eloy P, Poirrier AL. French adaptation and validation of the sino-nasal outcome test-22: a prospective cohort study on quality of life among 422 subjects. Clin Otolaryngol . 2015; 40(1):29-35. doi: 10.1111/coa.12315.

10. Piccirillo JF, Merritt MG Jr, Richards ML. Psychometric and clinimetric validity of the 20-Item SinoNasal Outcome Test (SNOT-20). Otolaryngol Head Neck Surg . 2002; 126(1):41-7. 
11. Mattos JL, Edwards C, Schlosser RJ, Hyer M, Mace JC, Smith TL, Soler ZM. A brief version of the questionnaire of olfactory disorders in patients with chronic rhinosinusitis.Int Forum Allergy Rhinol . 2019; 9(10):1144-1150. doi: 10.1002/alr.22392.

12. Bhattacharyya N, Kepnes LJ. Contemporary assessment of the prevalence of smell and taste problems in adults.Laryngoscope . 2015; 125:1102-6. doi: 10.1002/lary.24999.

13. Hummel, T., Kobal, G., Gudziol, H. et al. Normative data for the "Sniffin' Sticks" including tests of odor identification, odor discrimination, and olfactory thresholds: an upgrade based on a group of more than 3,000 subjects. Eur Arch Otorhinolaryngol 2007; 264:237-243. https://doi.org/10.1007/s00405-006-0173-0

14. Vaira LA, Deiana G, Fois AG, et al. Objective evaluation of anosmia and ageusia in COVID-19 patients: Single-center experience on 72 cases.Head Neck . 2020. doi: 10.1002/hed.26204.

15. Suzuki M, Saito K, Min WP, Vladau C, Toida K, Itoh H, Murakami S. Identification of viruses in patients with postviral olfactory dysfunction.Laryngoscope . 2007; 117(2):272-7.

16. Netland J, Meyerholz DK, Moore S, Cassell M, Perlman S.

Severe acute respiratory syndrome coronavirus infection causes neuronal death in the absence of encephalitis in mice transgenic for human ACE2. J Virol. 2008; 82:7264-75.

17. Li YC, Bai WZ, Hashikawa T. The neuroinvasive potential of SARS-CoV2 may play a role in the respiratory failure of COVID-19patients. J Med Virol . 2020. doi: 10.1002/jmv.25728.

Table 1: Epidemiological Characteristics of Patients.

\begin{tabular}{ll}
\hline Characteristic & All patients \\
\hline & $(\mathrm{N}=86)$ \\
Age & \\
Mean (SD) - yo & $41.7 \pm 11.8$ \\
Gender (N - \%) & \\
Female & $56(65.1)$ \\
Male & $30(34.9)$ \\
Ethnicity (N - \%) & \\
Caucasian & $84(97.7)$ \\
North African & $2(2.3)$ \\
Addictions (N - \%) & \\
Non-smoker & $77(89.5)$ \\
Mild smoker (1-10 cigarettes daily) & $7(8.1)$ \\
Moderate smoker (11-20 cigarettes daily) & $1(1.2)$ \\
Heavy smoker (>20 cigarettes daily) & $1(1.2)$ \\
Allergic patients & $16(18.6)$ \\
Comorbidities & \\
GERD & $9(10.5)$ \\
Asthma & $5(5.8)$ \\
Allergic rhinitis & $5(5.8)$ \\
Hypertension & $4(4.7)$ \\
Hypothyroidism & $3(3.5)$ \\
Psoriasis & $2(2.4)$ \\
Depression & $2(2.3)$ \\
Sarcoidosis & $1(1.2)$ \\
Hemochromatosis & $1(1.2)$ \\
Obstructive apnea syndrome & $1(1.2)$ \\
Autoimmune disease & $0(0)$ \\
&
\end{tabular}




\begin{tabular}{ll}
\hline Characteristic & All patients \\
\hline Diabetes & $0(0)$ \\
Renal failure & $0(0)$ \\
Hepatic insufficiency & $0(0)$ \\
Respiratory insufficiency & $0(0)$ \\
Heart problems & $0(0)$ \\
Neurological diseases & $0(0)$ \\
\hline
\end{tabular}

Table 1 footnotes : The mean PCR cycle number inversely reflects the viral load, According to the threshold of our Lab, 29 patients were positive for COVID-19 10 days (mean) after the initial diagnosis. Abbreviations: GERD=gastroesophageal reflux disease; $\mathrm{SD}=$ standard deviation.

Table 2: Severity of General Symptoms developed over the Clinical Course of the Disease (Percent of patients).

\begin{tabular}{llllll}
\hline General Symptoms & $0=$ No & 1=Very Mild & 2=Mild or & 3=Moderate & 4=Severe \\
\hline & Problem & Problem & Slight Problem & Problem & Problem \\
Fever & $64(91.4)$ & $5(7.1)$ & $1(1.4)$ & $0(0)$ & $0(0)$ \\
Cough & $36(51.4)$ & $18(25.7)$ & $12(17.1)$ & $4(5.7)$ & $0(0)$ \\
Chest pain & $57(81.4)$ & $6(8.6)$ & $5(7.1)$ & $1(1.4)$ & $1(1.4)$ \\
Loss of appetite & $43(61.4)$ & $10(14.3)$ & $8(11.4)$ & $5(7.1)$ & $4(5.7)$ \\
Sticky Sputum & $52(73.4)$ & $11(15.7)$ & $4(5.7)$ & $1(1.4)$ & $2(2.9)$ \\
Arthralgia & $48(68.6)$ & $8(11.4)$ & $5(7.1)$ & $7(10.0)$ & $2(2.9)$ \\
Myalgia & $40(57.1)$ & $19(27.1)$ & $3(4.3)$ & $6(8.6)$ & $2(2.9)$ \\
Diarrhea & $48(68.9)$ & $15(21.4)$ & $4(5.7)$ & $2(2.9)$ & $1(1.4)$ \\
Abdominal pain & $58(82.9)$ & $8(11.4)$ & $4(5.7)$ & $0(0)$ & $0(0)$ \\
Nausea/vomitting & $61(87.1)$ & $7(10.0)$ & $2(2.9)$ & $0(0)$ & $0(0)$ \\
Headache & $28(40.0)$ & $17(24.3)$ & $14(20.0)$ & $10(14.3)$ & $1(1.4)$ \\
Asthenia & $19(27.1)$ & $17(24.3)$ & $14(20.0)$ & $13(18.6)$ & $7(10.0)$ \\
Urticaria & $61(87.1)$ & $3(4.3)$ & $4(5.7)$ & $1(1.4)$ & $1(1.4)$ \\
Conjonctivitis & $52(74.3)$ & $12(17.1)$ & $2(2.9)$ & $3(4.3)$ & $1(1.4)$ \\
\hline
\end{tabular}

Table 2 footnotes: The symptoms severity was assessed with a 4-point scale (from no problem (0) to Severe problem (4)). The symptom data were available for 70 patients. The rest of the patients fulfilled the patient-reported outcome questionnaire a few days after the sniffin'stick tests, which may bias the analysis. The data of these patients were not considered in this table.

Table 3: Severity of Ear, Nose, and Throat Symptoms developed over the Clinical Course of the Disease (Percent of patients).

\begin{tabular}{llllll}
\hline Ear, nose \& throat & $0=$ No & 1=Very Mild & 2=Mild or & $3=$ Moderate & 4=Severe \\
\hline Symptoms & Problem & Problem & Slight Problem & Problem & Problem \\
Nasal obstruction & $29(38.6)$ & $23(32.9)$ & $12(17.1)$ & $5(7.1)$ & $1(1.4)$ \\
Rhinorrhea & $37(50.0)$ & $19(27.1)$ & $12(17.1)$ & $2(2.9)$ & $0(0)$ \\
Postnasal drip & $36(48.6)$ & $17(24.3)$ & $12(17.1)$ & $5(7.1)$ & $0(0)$ \\
Throat pain & $52(72.9)$ & $12(17.1)$ & $3(4.3)$ & $3(4.3)$ & $0(0)$ \\
Facial pain & $55(77.1)$ & $7(10.0)$ & $7(10.0)$ & $1(1.4)$ & $0(0)$ \\
Ear pain & $47(65.7)$ & $19(27.1)$ & $2(2.9)$ & $2(2.9)$ & $0(0)$ \\
Dysphagia & $63(88.6)$ & $2(2.9)$ & $4(5.7)$ & $1(1.4)$ & $0(0)$
\end{tabular}




\begin{tabular}{llllll}
\hline Ear, nose \& throat & $0=$ No & 1=Very Mild & 2=Mild or & $3=$ Moderate & $4=$ Severe \\
\hline Dyspnea & $52(72.9)$ & $12(17.1)$ & $3(4.3)$ & $3(4.3)$ & $0(0)$ \\
Dysphonia & $53(75.7)$ & $12(17.1)$ & $2(2.9)$ & $2(2.9)$ & $1(1.4)$ \\
Dysgeusia & $37(52.9)$ & $4(5.7)$ & $1(1.4)$ & $7(10.0)$ & $21(30.0)$ \\
\hline
\end{tabular}

Table 3 footnotes: The symptoms severity was assessed with a 4-point scale (from no problem (0) to Severe problem (4)). The symptom data were available for 70 patients. The rest of the patients fulfilled the patient-reported outcome questionnaire a few days after the sniffin'stick tests, which may bias the analysis. The data of these patients were not considered in this table.

Table 4: Sino-nasal Complaints of Patients with Olfactory Dysfunction.

\begin{tabular}{ll}
\hline SNOT-22 items & Mean $\pm(\mathrm{SD})$ \\
\hline Need to blow nose & $1.7 \pm 1.3$ \\
Nasal blockage & $1.1 \pm 1.1$ \\
Sneezing & $1.6 \pm 1.4$ \\
Runny nose & $1.6 \pm 1.3$ \\
Cough & $1.3 \pm 1.4$ \\
Post-nasal discharge & $0.7 \pm 1.0$ \\
Thick nasal discharge & $0.6 \pm 1.1$ \\
Ear fullness & $0.6 \pm 1.1$ \\
Dizziness & $0.7 \pm 1.1$ \\
Ear pain & $0.6 \pm 1.0$ \\
Facial pain/pressure & $0.9 \pm 1.3$ \\
Decreased sense of smell/taste & $1.2 \pm 1.6$ \\
Difficulty falling asleep & $1.6 \pm 1.7$ \\
Wake up at night & $1.8 \pm 1.7$ \\
Lack of a good night's sleep & $2.1 \pm 1.7$ \\
Wake up tired & $2.4 \pm 1.6$ \\
Fatigue & $1.9 \pm 1.5$ \\
Reduced productivity & $1.8 \pm 1.6$ \\
Reduced concentration & $1.7 \pm 1.6$ \\
Frusated/restless/irritable & $1.6 \pm 1.5$ \\
Sad Embarrassed & $4.2 \pm 1.3$ \\
SNOT-22 total score & $1.8 \pm 1.4$ \\
Short version QOD-NS items & $33.3 \pm 19.0$ \\
Changes in my sense of smell isolate me socially. & \\
The problems with my sense of smell have a negative impact on my daily social activities & $1.8 \pm 0.9$ \\
The problems with my sense of smell make me more irritable & $1.7 \pm 1.0$ \\
Because of the problems with my sense of smell, I eat out less & $1.4 \pm 1.2$ \\
Because of the problems with my sense of smell, I eat less than before (loss of appetite) & $1.3 \pm 1.1$ \\
Because of the problems with my sense of smell, I have to make more effort to relax & $2.0 \pm 0.8$ \\
I'm afraid I'll never be able to get used to the problems with my sense of smell. & $1.1 \pm 1.0$ \\
Short version QOD-NOS total score & $10.3 \pm 5.7$ \\
\hline
\end{tabular}

Table 4 footnotes : Abbreviations: QOD-NS= short version of Questionnaire of Olfactory DisordersNegative Statements; SNOT-22= sinonasal outcome test-22.

Table 5: Characteristics of Anosmic, Normosmic and Hyposmic Patients . 


\begin{tabular}{|c|c|c|c|c|}
\hline & Anosmic $(\mathrm{N}=41)$ & Hyposmia $(\mathrm{N}=12)$ & Normosmia $(\mathrm{N}=33)$ & $\mathrm{p}$-value \\
\hline Age - (Mean, SD) & $40 \pm 12$ & $39 \pm 13$ & $45 \pm 11$ & NS \\
\hline $\operatorname{Sex}(M / F)$ & $15 / 36$ & $3 / 0$ & $12 / 21$ & NS \\
\hline Tabacco (yes/no) & $5 / 36$ & $2 / 10$ & $2 / 31$ & NS \\
\hline \multicolumn{5}{|l|}{ Comorbidities (yes/no) } \\
\hline Hypertension & 0 & 2 & 2 & 0.027 \\
\hline Rhinitis & 1 & 2 & 2 & NS \\
\hline Reflux & 4 & 1 & 4 & NS \\
\hline Asthma & 2 & 2 & 1 & NS \\
\hline SNOT-22 (Mean, SD) & $33 \pm 16$ & $43 \pm 20$ & $34 \pm 19$ & NS \\
\hline Nasal obstruction (yes/no/NC) & $13 / 20 / 8$ & $7 / 2 / 3$ & $18 / 10 / 5$ & NS \\
\hline Self-reported Total loss of smell (N/\%) & $26(78.8)$ & $8(88.9)$ & $18(64.3)$ & NS \\
\hline Duration of Anosmia (Mean, SD - days) & $17 \pm 11$ & $18 \pm 11$ & $17 \pm 10$ & NS \\
\hline
\end{tabular}

Table 5 footnotes : All 86 patients performed Sniffin'Stick tests. However, only 70 patients completed the two questionnaires (general and SNOT-22) the same day of the olfactory test. The patient questionnaires, which were fulfilled after the olfactory dysfunction, were not considered regarding the risk of bias (NC in the table). Abbreviations: $\mathrm{M} / \mathrm{F}=$ male/female; $\mathrm{NC}=$ not considered; $\mathrm{SD}=$ standard deviation.

Figure 1: Chart flow 


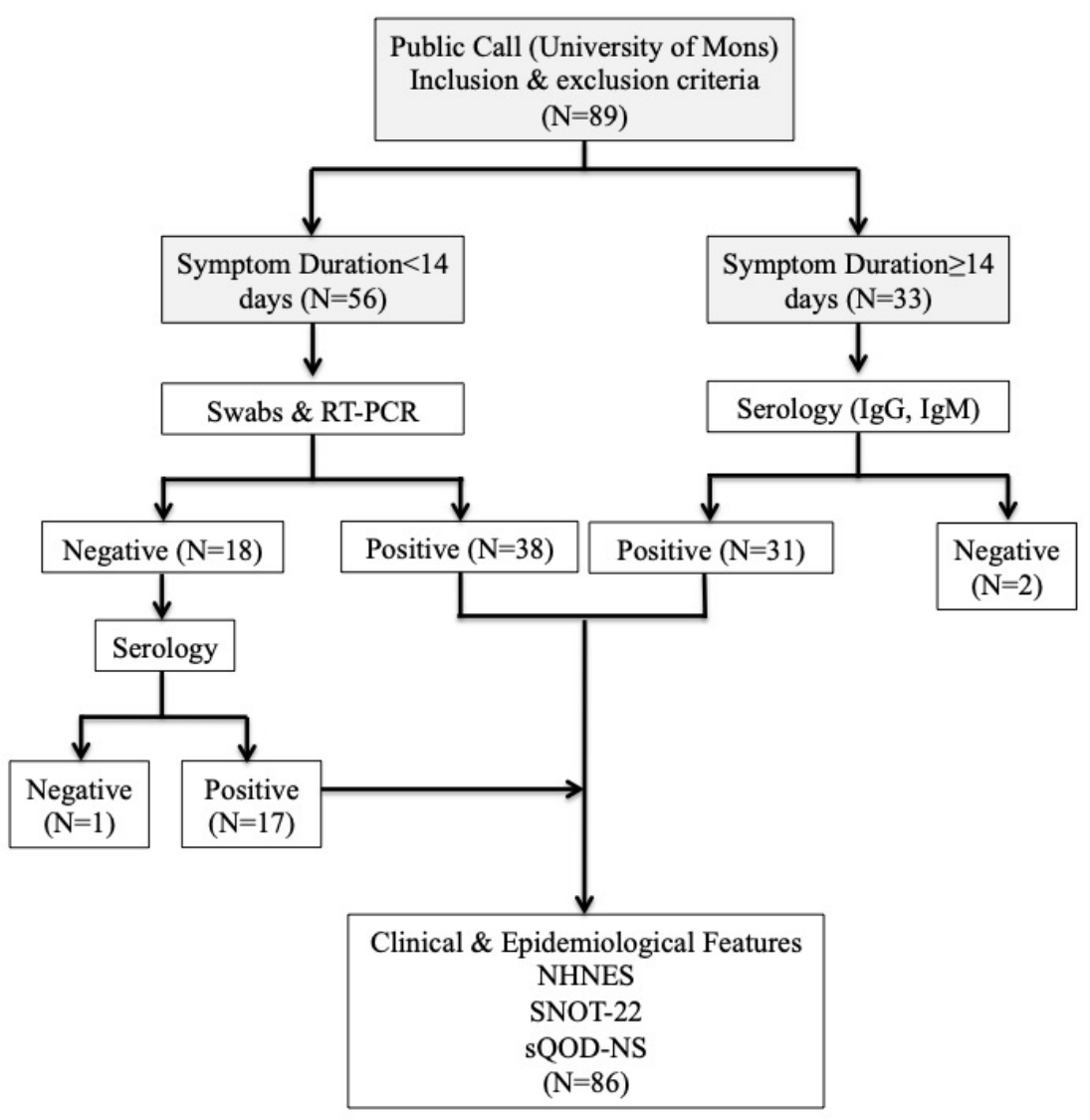

Figure 1: Abbreviations: NHNES: National Health and Nutrition Examination Survey; SNOT-22: Sinonasal outcome tool-22; sQOD-NS: short version of Questionnaire of Olfactory Disorders-Negative Statements. 